\title{
Forestry Technician Program Gold Medal Winners
}

\section{Sault College - Wendy Lambert}

This year's recipient of the CIF Gold Medal Award is Wendy Lambert.

Wendy commenced her studies in January 1993 and graduated with honours in December 1994 from the Forestry Technician Program. She earned the respect of both her classmates and her teachers while at Sault College. She was

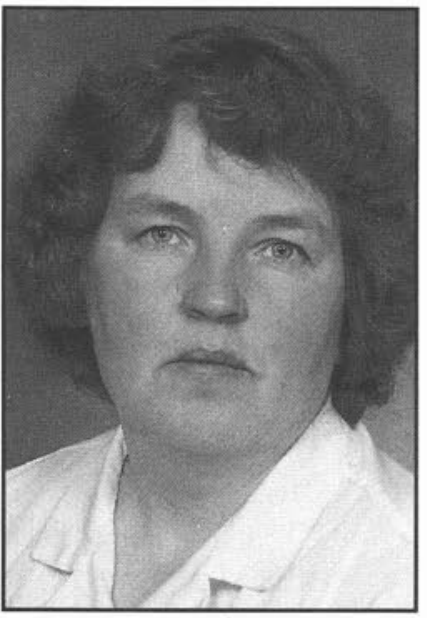
a joy to teach and was always willing to help out her classmates. Wendy worked in the Growth \& Yield program for the past 2 summers and is looking forward to working there again this summer.

\section{Cégep de Baie-Comeau - Mme Brigitte Courtois}

Mme Brigitte Courtois effectuait en 1993 un retour au Cégep après avoir fait un cheminement d'études et de travail dans le domaine des communications, comme traductrice pour Bell Canada. Son choix pour le domaine forestier constitue un changement complet de carrière et de discipline. Mme Courtois s'était fixée comme objectif de développer son sens pratique par l'apprentissage

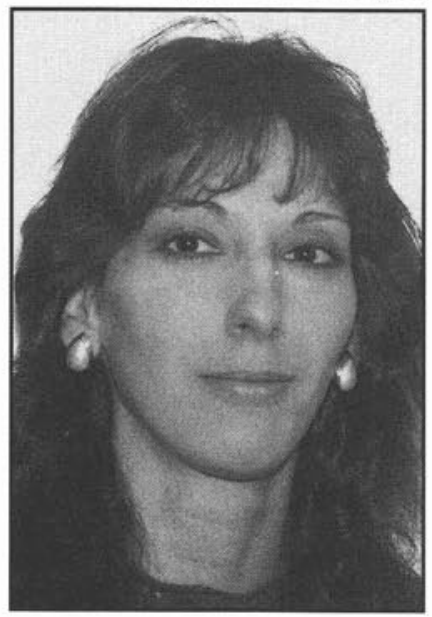
d'une technique tout en évoluant harmonieusement dans un milieu majoritairement composé d'hommes. Elle a su développer son sens de l'organisation et son goût des responsabilités. Cependant, sa formation en lettres refait continuellement surface car elle réserve toujours une place importante au français dans sa vie professionnelle.

\section{Cégep de Rimouski - Serge Racine}

La motivation et la volonté d'apprendre sont directement reliées à l'intérêt porté à la discipline étudiée. Cette phrase explique à elle seule les raisons pour lesquelles je suis retourné étudier en technologie forestière à l'âge de 28 ans. Trois années au cours desquelles les découvertes, les apprentissages et les habiletés se sont succédés. Tout cet enseignement s'est appliqué concrètement lors de stages d'été: le premier, à la forêt d'enseignement et de recherche de mon institution scolaire à

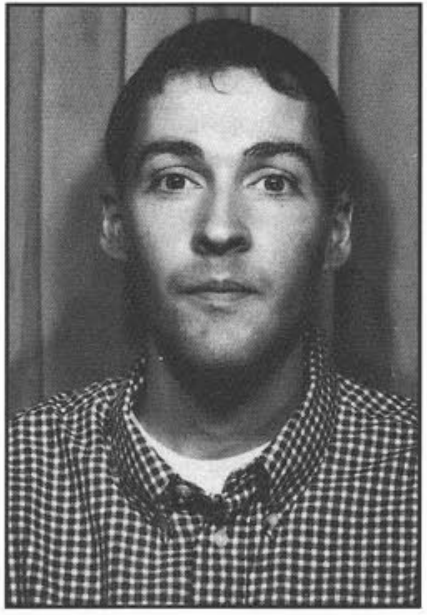
Rimouski; le second, dans divers organismes de Haute-Savoie en France.

Fortement intéressé par l'approche de la gestion intégrée des ressources et par la géomatique, je souhaite pouvoir prendre part à la mise en valeur de la forêt d'aujourd'hui afin qu'elle puisse être encore présente demain.

\section{Environmental Training Centre - Marcie Giroux}

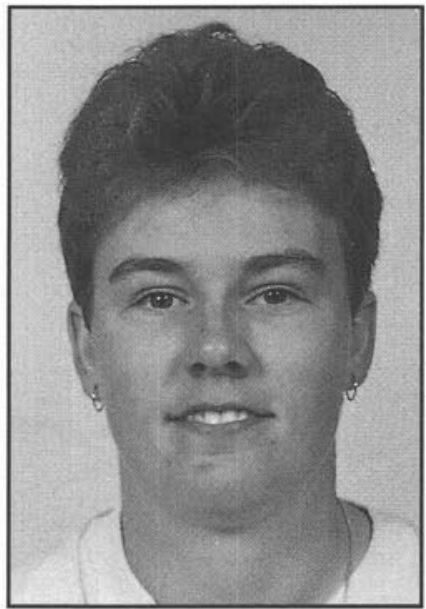

The recipient of the CIF Gold Medal for 1995 at the Environmental Training Centre is Marcie Giroux. Marcie has attained the top academic standing in the NAIT two year Forest Technology program for two consecutive years. Marcie attended high school in Calgary. During her two year program at NAIT in Edmonton and at ETC in Hinton, Marcie served as treasurer of the student council.

During the summer of 1994 she worked with Pinewood Forestry Services in Grande Prairie conducting various silvicultural surveys. Upon graduation Marcie will be employed by Canfor with the Hines Creek Division as a silvicultural technologist.

Curling is one sport she participated in during her spare time. While at Hinton she and a classmate were instrumental in establishing a Venturer Company in the scouting organization. This Company will be passed on to other leaders as Marcie leaves for her new job.

\section{CIF/IFC Membership Dues 1995-1996}

\section{Active Member}

1st year after graduation (Grad. 1995) (\$1.31 GST incl.) 2nd year after graduation (Grad. 1994) (\$2.49 GST incl.) Other new members for first year ( $\$ 2.49 \mathrm{GST}$ incl.)

Married or equivalent, second member only ( $\$ 2.49$ GST incl.) All other active members ( $\$ 2.49$ GST incl.)
$\$ 21.00$

$\$ 93.00$

$\$ 93.00$

$\$ 93.00$

$\$ 131.00$
Other Memberships

Retired (must have more than 15 years CIF/IFC membership) (\$2.49 GST incl.) $\$ 42.00$

Student ( $\$ 1.31$ GST incl.)

$\$ 21.00$

Sustaining individual

$\$ 44.00$

Sustaining corporate (\$4.98 GST incl.) $\$ 275.00$ 